\title{
A network pharmacological approach to evaluate Jia-Wei-Xiao-Yao-san formula's mechanistic pathways and its implication in the symptomatology of polycystic ovarian syndrome
}

\begin{abstract}
Polycystic ovary syndrome (PCOS) is a disorder characterized mainly by a disruption of androgen secretion or endocrine feedback mechanism dysfunction occurring subtlety during adolescence and progressively impairing the ovarian folliculogenesis which affects preponderantly women of reproductively active age. Most of the reproductive active period of women is hampered by this condition, thereof reducing their fertility and causing metabolic disturbances. Traditional Chinese Medicine principles have been historically settled to develop Chinese Herbal Medicine formulas designed to treat various ailments including reproductive disorders. This study addresses the mechanisms of action of JiaWei-Xiao-Yao-San formula by examining the functional predicted PCOS-related targets networks first enriched by STITCH chemical interactions and then analyzed by STRING protein interactions database. Using the highest confidence interaction score as a screening binding parameter, we obtained a total of 274 PCOS-related targets interacting with 58 bioactive compounds from the formula and 22 PCOS-related drugs interacting with a total of 133 PCOS-related targets. Our analysis identified a total of 84 common PCOSrelated targets shared by both the formula and the PCOS-related drugs and demonstrate the predicted PCOS-related protein-protein interaction network resulting in various KEGG pathways. These findings indicated the formula functionality role preponderantly in cholesterol metabolism, ovarian steroidogenesis, peroxisome proliferator-activated receptors, and adipocytokine signaling pathway. Numerous compounds identified here are known to have high binding interactions with PCOS-related targets. Our methodological approach demonstrates that both the drugs-PCOS-related targets network support Jia-WeiXiao-Yao-San formula as an appropriate Traditional Chinese Medicine to treat at least the symptoms associated with PCOS manifestations.
\end{abstract}

Keywords: traditional herbal medicine, compounds-targets network, PCOS-related genes, signaling pathways, ovarian steroidogenesis
Volume 13 Issue 5 - 2020

\author{
Benoit Leclerc, ${ }^{1,2}$ Yu Chu Wu, ${ }^{3}$ Priscilla Wu ${ }^{2,3}$ \\ 'Department of Research \& development, MatrixHerbs \\ Research Institute, Canada \\ ${ }^{2}$ Consultant in TCM, MatrixHerbs Biotechnology Inc, Canada \\ ${ }^{3}$ Management, Fertility Online Clinic, Canada
}

Correspondence: Benoit Leclerc at the MatrixHerbs Biotechnology Inc., 65 Sainte-Therese Street, Gatineau, Quebec, J9A 2N5, Canada, Tel I-888-844-9838, Fax I-833-444-9838, Emailinfo@matrixherbsbiotech.com

Received: October 03, 2020 | Published: October 19, 2020

\begin{abstract}
Abbreviations: CHM, Chinese herbal medicine; CT, compounds-targets network; CDT, Compounds-drugs-targets network; CYP19A1, cytochrome P450 family 19 subfamily A member 1; CYP17A1, cytochrome P450 family 17 subfamily A member 1; DHEAS, dehydroepiandrosterone sulfate; DT, Drugs-targets network; F2R, coagulation factor 2 receptor; FSH, Follicle-stimulating hormone; FSHR, follicle-stimulating hormone receptor; GnRH, gonadotropin-releasing hormone; JWXYS, Jia-Wei-Xiao-Yao-San; KEGG, Kyoto encyclopedia of genes and genome; LH, luteinizing hormone; LHCGR, luteinizing hormone/choriogonadotropin receptor; PCOS, polycystic ovarian syndrome; SHGB, sex hormone-binding globulin; TCM, traditional chinese medicine; THM, traditional herbal medicine; WM, western medicine
\end{abstract}

\section{Introduction}

Polycystic ovarian syndrome (PCOS) is a condition that progressively develops over a reproductive-aged population of women trying to conceive a child. Recent meta-systemic analyses pointed out at large trials variation (5-13\%) in the overall prevalence of PCOS, according to the National Institutes of Health, Rotterdam and Androgen Excess Society criteria. ${ }^{1-3}$ These variations are due to problematic definitions of the PCOS syndrome and its phenotypic expression (e.g. hyperandrogenism to hirsutism, anovulation to infertility, amenorrhea to irregular/heavy uterine bleeding, insulin resistance to obesity), as well as its diagnostic cut-off between different study size cohorts and ethnicities in an unselected population, and these criteria contribute in patients' PCOS diagnosis bias. ${ }^{1-4}$ Hence, identifying the correct PCOS etiology is pivotal in adopting the best clinical practices.

Three theories have been established to support the PCOS etiology and its pathophysiology. The first group of women who develop PCOS experiences a disordered hypothalamic gonadotropin secretion which results in a higher pituitary LH and a lower FSH secretion compared to age and weight-matched women without PCOS.., 6 Since the hypothalamic gonadotropin-releasing hormone (GnRH) becomes insensitive to progesterone feedback mechanism during the earlier years of active reproduction, thecal cells exposed to high luteinizing hormone (LH) stimulation can only produce androgens while granulosa cells exposed to low follicle-stimulating hormone (FSH) level produce inadequate levels of androgens aromatization which reduces conversion to estrogens. ${ }^{7,8}$ The imbalance of androgens to estrogens ratio can cause 1) abnormal ovarian morphology by generating excessive thecal and stromal hyperplasia within the 
ovaries, 2) hirsutism by stimulating the sebum production of the pilosebaceous unit leading to hair growth, and 3) anovulation by blocking the pre-antral follicles stage which accumulates in one/both ovaries. ${ }^{4,8-10}$ To the end, a vicious feedback mechanism is gradually taking place where an excess of LH leads to higher ovarian androgens production which further stimulates pituitary LH secretion. ${ }^{8}$. The second group of women develops PCOS because their primary ovarian steroidogenesis pathway is perturbed along with higher circulating androgen levels resulting in the same PCOS phenotypic effect (e.g. hirsutism) as those with a primary disordered gonadotropin secretion. ${ }^{11}$ A proportion of $20-30 \%$ of women with PCOS has adrenal hyperandrogenism suggesting a primary steroidogenesis defect which augments the dehydroepiandrosterone sulfate (DHEAS) levels and therefore affects the androgen-secreting ovary and adrenal gland. ${ }^{8,12,13}$ Other factors indicated that some women could have a decrease loss of oocytes during childhood, puberty, and early adulthood increasing the density of small preantral follicles in polycystic ovaries. ${ }^{8,14}$ The third group of women develops PCOS as a primary disorder of insulin resistance. From early to later ontogeny of the syndrome, there is a progressive increased pancreatic beta cell production of insulin and dyssynchronous pulse between fasting and postprandial hyperglycemia in these women..$^{15-18}$ Additionally, women developing hyperinsulinemia associated with PCOS reflect the ability of insulin to perturb ovaries and adrenals androgen production. ${ }^{19,20}$ Thus, insulin stimulates gonadal and adrenal androgen secretion which, in turn, allows androgens to stimulate insulin resistance via decreasing sex hormone-binding globulin (SHBG). ${ }^{21}$ The evolution of PCOS can result in insulin resistance with the compensation mechanism of hyperinsulinemia associated with obesity which could lower the SHBG leading to higher levels of free testosterone and other abnormal levels of sex hormones levels that can elongate menstrual cycles. ${ }^{8,22,23}$ About $50 \%$ of all women with PCOS are overweight or obese; they are at a higher risk of developing non-alcoholic fatty acid liver. ${ }^{24,25}$ Also, these women are likely of developing metabolic syndrome which encompasses higher blood pressure, abdominal fat gain, changes in blood lipoprotein composition, and higher blood glucose levels. ${ }^{26,27}$ About 20\% of women with PCOS are more likely to experience sleep apnea and depression than those in the unselected population..$^{28-31}$

Traditional Chinese medicine (TCM) principles have been historically settled to develop Chinese herbal medicine (CHM) formulas designed to treat various ailments. Nowadays, women with PCOS can be offered with western medicine (WM), e.g. clomiphene citrate used to treat an ovulation. Besides, PCOS women can be prescribed with CHM only or a combination of WM and CHM. Deciphering the biological effects of numerous CHMs related to human illnesses is an intense area of research to provide effective therapies either for improving alternative medicine or medical intervention. In this study, we examined the molecular and biological basis of Jia-WeiXiao-Yao-San formula (JWXYS) using pharmacology network tools to analyze one of the most commonly used herbal formulas prescribed for PCOS suffering Asian women.

\section{Material and methods}

\section{Jia-Wei-Xiao-Yao-San formula composition and its TCM actions}

Various modifications of the original JWXYS have been documented based on the patients' condition and severity, the specific targeted disease, and other factors involved such as age, history of the medical condition, health complication, and current medications are taken. According to the https://www.americandragon.com/, http:// www.tcmip.cn/ETCM/index.php/Home/Index/, https://kampo.ca/, Chinese Materia Medica, ${ }^{32}$ Jia-Wei-Xiao-Yao-San formula (JWXYS) is generally composed of the following ingredients: angelicae sinensis radix, poria, gardenia fructus, menthae haplocalycis herba, paeoniae radix alba, bupleuri radix, glycyrrhizae radix et rhizome, atractylodis macrocephalae rhizome, moutan cortex, and zingiberis rhizome recens. The JWXYS formula herbal composition and its TCM principles are depicted in Figure 1. When preparing a CHM formula prescription, it is essential to know the dosage composition of the original formula to adjust it under the overall patients' health conditions with their corresponding specifics factors. According to the original JWXYS,${ }^{33-36}$ the following dosage ingredients are angelicae sinensis radix $(1 \mathrm{~g})$, poria $(1 \mathrm{~g})$, gardenia fructus $(0.67 \mathrm{~g})$, menthae haplocalycis herba $(0.33 \mathrm{~g})$, paeoniae radix alba $(1 \mathrm{~g})$, bupleuri radix $(1 \mathrm{~g})$, glycyrrhizae radix et rhizome $(0.5 \mathrm{~g})$, atractylodis macrocephalae rhizome $(1 \mathrm{~g})$, moutan cortex $(0.67 \mathrm{~g})$, and zingiberis rhizome recens $(0.33 \mathrm{~g})$ (Figure 1)

\section{Jia-Wei-Xiao-Yao-San formula indications}

According to population-based studies conducted in Taiwan, patients with uterine bleeding or hypertension received a JWXYS average daily dose of 7.3-7.5g. ${ }^{37,38}$ Previous studies pointed out the indications JWXYS to be especially effective for alleviating menopausal symptoms and menstrual disorders..$^{33,39,40-42}$ A clinical study indicated the use of JWXYS for improving depression status in menopause patients by increasing serum levels of TNF-alpha after 12 weeks of treatment ${ }^{43}$ and supported by a retrospective populationbased study that showed JWXYS as the most prescribed formula (12\%) for patients having sleep disorders and major depressive disorder. ${ }^{44} \mathrm{~A}$ pharmacological-epidemiological study revealed that JWXYS was the most commonly used formula (38\%) for alleviating premenstrual syndrome with an average dose of $4.9 \mathrm{~g} .{ }^{45}$ Besides, PCOS patients were prescribed with the most popular JWXYS formula (38\%) using an average daily dose of $6.1 \mathrm{~g} .^{46}$

\section{Computational methodology and networks analysis}

We have identified a total of 811 chemical compounds in the JWXYS formula from the Traditional Chinese Medicines Integrated Database (www.megabionet.org/tcmid) and a total of 1424 targets associated with the polycystic ovarian syndrome (PCOS) condition (https://www.targetvalidation.org). Compounds-PCOS targets networks were analyzed by the STITCH chemicals interactions database (http://stitch.embl.de) for assessing the predicted proteins network. Subsequently, the functional predicted clustering networks were first enriched and then analyzed by the STRING proteins interactions database (https://string-db.org) to retrieve each clustering networks' biological processes, molecular functions, and Kyoto Encyclopedia of Genes and Genomes (KEGG) pathways. The top signaling pathways were interpreted according to the phenotypic group of all PCOS conditions. Using STITCH, we selected a total of 274 PCOS targets having the highest interacting score at 0.9 with 58 out of 142 bioactive compounds in JWXYS formula is shown in Table 1. Next, we were interested in knowing the phenotypic groups among PCOS patients as their diagnoses are often misinterpreted by doctors and drug therapies given to these patients contribute to decreasing the medical intervention success rate. Using drugs as a benchmark, we searched in PCOS-related drug databases (https://www.drugbank. ca, https://www.clinicaltrials.gov, ${ }^{47}$ to compare the drugs associated 
with PCOS-related targets with the formula compounds binding to the PCOS-related targets and finding common targets. To do so, we identified a total of 22 selective drugs (e.g. alogliptin, chorionic gonadotropin, clomiphene citrate, cyproterone acetate, desogestrel, diazoxide, dydrogesterone, ethynodiol diacetate, ethynylestradiol, finasteride, flutamide, letrozole, levonorgestrel, mestranol, metformin, moxonidine, myo-inositol, orlistat, pioglitazone, rosiglitazone, sibutramine \& spironolactone) that have the highest interaction score with a total of 133 PCOS-related targets following STITCH analysis. We identified a total of 84 common targets that belong to PCOS- related formula and PCOS-related drugs and then address the common PCOS-related targets involved in KEGG pathways. Besides, we found a total of 190 and 49 unique targets from PCOS-related formula and PCOS-related drugs, respectively. The methodological approach, as shown in Figure 2, allowed us to determine the relationship between compounds-PCOS targets-pathways (CT network) and drugsPCOS targets-pathways (DT network) by constructing a common compounds-drugs-PCOS-related targets (CDT network) where the subsequent STRING clustering analyses were performed on CDT network.

DANG GUI (Angelicae sinensis radix)

Tonify blood, activate blood and regulate menstruation to relieve pain, moisten

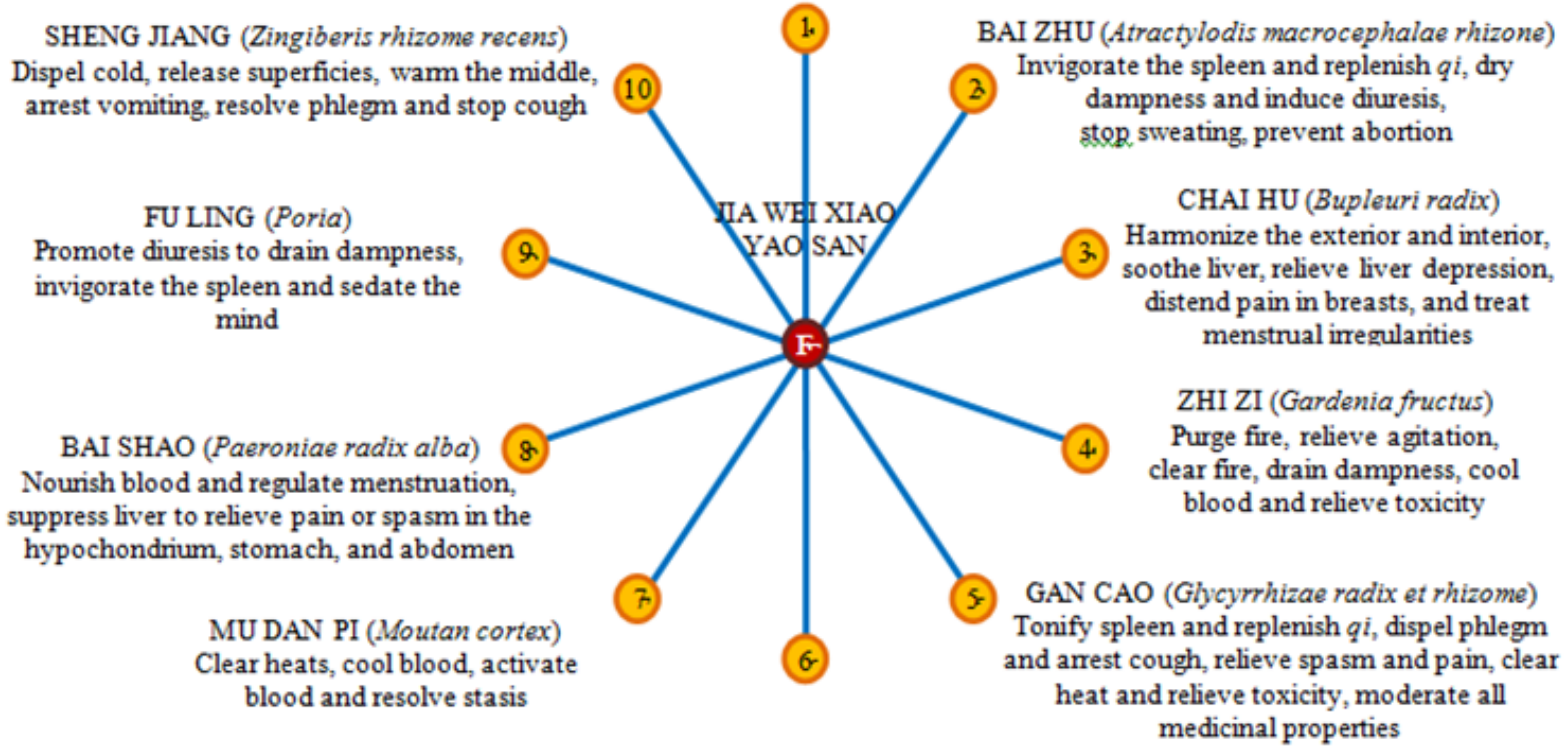

BO HE (Menthae haplocalycis herba)

Disperse wind-heat, clear head and eyes, soothe throat, promote enuption, soothe liver and regulate

qi

Figure I List of single herbs composing JWXYS formula and their respective medicinal actions based on the TCM concepts. Text in bold refers to actions emphasized within the formula. Herbs interactions, not illustrated here for simplicity, are multiples, bidirectional, and underlie distinct types of combination involving for example mutual reinforcement, mutual assistance, and mutual restraint to alleviate PCOS condition. The letter F is denoted as the jia-Wei-XiaoYao-San formula.

Table I List of the JWXYS formula compounds having the highest interactions score with PCOS targets

\begin{tabular}{lll}
\hline Compound CID & Compound name & Name of the ingredients \\
\hline 5280442 & Acacetin & Menthae Haplocalycis Herba \\
177 & Acetaldehyde & Zingiberis Rhizoma Recens \\
175 & Acetate & Paeoniae Radix Alba \\
190 & Adenine & Angelicae Sinensis Radix \& Poria \\
60961 & Adenosine & Bupleuri Radix \\
5950 & Alanine & Menthae Haplocalycis Herba \\
7478 & Anisic acid & Angelicae Sinensis Radix \\
\hline
\end{tabular}

Citation: Leclerc B,Wu YC,Wu P.A network pharmacological approach to evaluate Jia-Wei-Xiao-Yao-san formula's mechanistic pathways and its implication in the symptomatology of polycystic ovarian syndrome. Int J Complement Alt Med. 2020;13(5):200-213. DOI: I0.15406/ijcam.2020.13.005I7 


\begin{tabular}{|c|c|c|}
\hline Compound CID & Compound name & Name of the ingredients \\
\hline 5280443 & Apigenin & $\begin{array}{l}\text { Gardeniae Fructus, Glycyrrhizae Radix et Rhizoma \& Menthae Haplocalycis } \\
\text { Herba }\end{array}$ \\
\hline 104775 & Butyrate & Angelicae Sinensis Radix \\
\hline 689043 & Caffeic acid & Menthae Haplocalycis Herba \\
\hline 2537 & Camphor & Angelicae Sinensis Radix \& Zingiberis Rhizoma Recens \\
\hline 1548943 & Capsaicin & Poria \& Bupleuri Radix \\
\hline 305 & Choline & Gardeniae Fructus, Angelicae Sinensis Radix \& Poria \\
\hline 4678093 & Decanoate & Angelicae Sinensis Radix \\
\hline 445070 & Farnesol & Zingiberis Rhizoma Recens \\
\hline 9651 & Galantamine & Glycyrrhizae Radix et Rhizoma \\
\hline 370 & Gallic acid & Moutan Cortex \\
\hline 5280961 & Genistein & Gardeniae Fructus \\
\hline 33032 & Glutamic acid & Menthae Haplocalycis Herba \\
\hline 10114 & Glycyrrhetinic acid & Glycyrrhizae Radix et Rhizoma \\
\hline 14982 & Glycyrrhizin & Glycyrrhizae Radix et Rhizoma \\
\hline 10621 & Hesperidin & Menthae Haplocalycis Herba \\
\hline 8892 & Hexanoic acid & Angelicae Sinensis Radix \\
\hline 5281654 & Isorhamnetin & Bupleuri Radix \\
\hline 3893 & Lauric acid & Bupleuri Radix, Poria \\
\hline I 35403648 & Leucovorin & Angelicae Sinensis Radix \\
\hline 5280934 & Linolenic acid & Bupleuri Radix \\
\hline 1176 & Lotion (synonym urea) & Glycyrrhizae Radix et Rhizoma \\
\hline 5280445 & Luteolin & Menthae Haplocalycis Herba \\
\hline 6287 & L-valine & Menthae Haplocalycis Herba \\
\hline 5962 & Lysine & Menthae Haplocalycis Herba \\
\hline 6251 & Mannitol & Gardeniae Fructus \\
\hline 342 & m-cresol & Angelicae Sinensis Radix \\
\hline 880 & Methylglyoxal & Glycyrrhizae Radix et Rhizoma \& Zingiberis Rhizoma Recens \\
\hline 892 & Myo-inositol & Gardeniae Fructus \\
\hline 11005 & Myristic acid & Angelicae Sinensis Radix \\
\hline 4276 & Myristicin & Angelicae Sinensis Radix \\
\hline 931 & Naphthalene & Angelicae Sinensis Radix \& Bupleuri Radix \\
\hline 938 & Niacin & Angelicae Sinensis Radix \\
\hline 454 & Octanal & Zingiberis Rhizoma Recens \\
\hline 119389 & Octanoate & Bupleuri Radix \& Zingiberis Rhizoma Recens \\
\hline 445639 & Oleic acid & Gardeniae Fructus \\
\hline 985 & Palmitate & Angelicae Sinensis Radix, Bupleuri Radix \& Poria \\
\hline
\end{tabular}




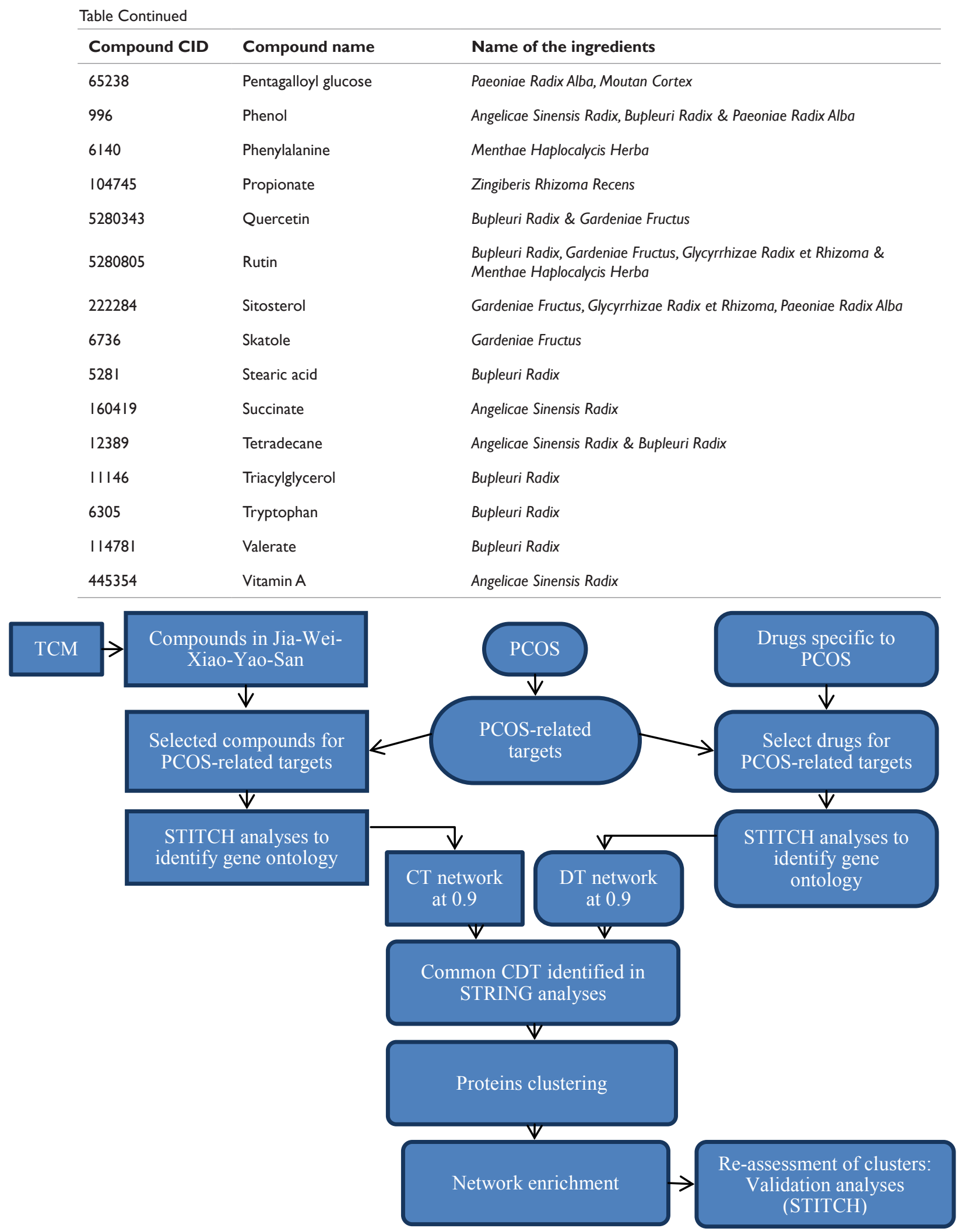

Figure 2 Diagram showing the pharmacology-based analysis of the JWXYS compounds to PCOS-related targets (CT), the drugs to PCOS-related targets (DT), and the common compounds to drugs to PCOS-related targets (CDT) network to assess the JWXYS formula biological effects. 
STITCH analyses identified the active compounds of the JWXYS formula having binding evidence at 0.7 , e.g. Atractylodis Macrocephalae Rhizoma and Moutan Cortex, and at 0.9 for the eight remaining ingredients towards the PCOS-related targets, therefore establishing a functional compounds-targets (CT) network assessment (Figure 3). Comparatively, the confidence interaction scores set at 0.7 and 0.9 of the DT networks were also evaluated as benchmark analyses. Clustering single herbs to PCOS-related targets were constructed using the Cytoscape software (version 3.8.0).

\section{Results}

\section{Selective criteria for Jia-Wei-Xiao-Yao-San formula}

Searching for the optimal THM formula in treating PCOS is a challenging task because it should meet essential characteristics. For instance, the formula should be well documented and reported in English peer-reviewed publications. Secondly, the formula should be clinically relevant in treating the symptoms of women with PCOS. Thirdly, the subject and quality of publications findings related to other human health conditions are important criteria to consider when deciding which THM formula to choose in the pharmacological analyses with PCOS-related targets. According to our analysis on the available THM formulas, we choose the Jia-Wei-Xiao-Yao-San formula as the most documented formula in regards to general health conditions and its popularity to treat symptoms associated with PCOS.

\section{Jia-Wei-Xiao-Yao-San bioactive compounds}

Following STITCH analyzing, the JWXYS formula has revealed a total of 58 out of 142 bioactive compounds interacting with the highest confidence score (0.9) to PCOS-related targets. Among the ingredients composing the formula, Angelicae Sinensis Radix has 17 compounds interacting with 172 PCOS-related targets, Bupleuri Radix has 16 compounds interacting with 180 PCOS-related targets, Gardeniae Fructus has 11 compounds interacting with 89 PCOSrelated targets, Menthae Haplocalycis Herba has 10 compounds interacting with 99 PCOS-related targets, Zingiberis Rhizoma Recens has 8 compounds interacting with 74 PCOS-related targets, Poria has 8 compounds interacting with 108 PCOS-related targets, Glycyrrhizae Radix et Rhizoma has 6 compounds interacting with 12 PCOS-related targets, and Paeoniae Radix Alba has 4 compounds interacting with 71 PCOS-related targets. Using an interaction score of 0.7, Moutan Cortex shows 5 compounds interacting with 17 PCOS-related targets and Atractylodis Macrocephalae Rhizoma has only 1 known compound interacting with 2 PCOS-related targets (Figure 3 ).

\section{TCM concepts related to Jia-Wei-Xiao-Yao-San formula}

The TCM concepts explain generally the medicinal actions of the overall JWXYS formula, such as tonifying blood and regulating menstruation, promoting diuresis, soothing liver, and qi, tonifying the spleen, relieving spasm and pain, and moderating all other ingredients properties within the formula, but it doesn't explain the molecular occurrence. Therefore, performing pharmacological analyses are essential to highlight the biological and molecular effects of the bioactive compounds found within the JWXYS formula. We built the JWXYS formula CT network using a 0.9 confidence score to assess its highest molecular binding interaction. Next, PCOS drugs were selected, and its DT network was built using the same confidence score as the JWXYS formula. Both networks were overlapped to select common PCOS-related targets shared by both (Figure 4A) which are clustered into functional proteins networks. These protein-protein interactions clusters are assigned to KEGG pathways and reassessed on the STITCH tool to validate the pharmacological-basis analysis. The 190 unique targets binding to the JWXYS formula compounds were evaluated, but not considered here due to the complexity of the findings. The TCM concepts associated with the JWXYS formula is important in modulating the circulatory vascular system along with regulating the liver and spleen while balancing the cold and heat can alleviate the PCOS symptoms. However, we did not try to associate TCM concepts to the molecular evidence identified by STITCH analyses.

\section{Compounds-drugs-PCOS-related targets clusters assigned to KEGG pathways}

Once the CDT network has been built, we searched for the interrelationship between the preponderance of PCOS-related targets found within each cluster ID (Figure 4B) to the assigned KEGG pathways. Based on our findings, red nodes represent the neuroactive ligand-receptor interaction with a negative $\log$ of -14.58 are mostly located in the first cluster ID (Figures 4B, 4C, 4D). A total of 19 red nodes are represented; among them, 14 red nodes are located in the first cluster ID (KISS1R, P2RY2, EDNRB, EDNRA, OXTR, F2R, PTGER1, CHRM3, TACR3, GHSR, GNRHR, HTR2C, AGTR1, LPAR3) composed of 33 nodes. A total of 14 blue nodes represent the calcium signaling pathway with a negative $\log$ of -11.26 and 10 blue nodes are found in the first cluster ID (GNAQ, PTGER1, EDNRB, EDNRA, OXTR, F2R, CHRM3, TACR3, HTR2C, AGTR1). Interestingly, 8 yellow nodes represent the ovarian steroidogenesis, considered the closest pathway related to PCOS. Having a negative log of -8.33 , the first set of three yellow nodes is located in the sixth cluster ID with only three nodes involving the cytochrome targets, and the second set of three yellow nodes is found in the third cluster-ID having 6 nodes showing interaction with FSHR, LHCGR, and INS. Besides, we observed 8 dark green nodes exclusively found in the second cluster-ID composed of 29 nodes. The cholesterol pathway having the same negative log as the previous pathway showed great interaction with apolipoproteins (APOE, APOB, APOA1) and LDLR, LIPC, LPL, CD36, ANGPTL4. The PPAR signaling pathway is composed of 8 pink nodes all within the second cluster ID with a negative log of -7.27. The interactive targets include FABP4, PPARG, RXRA, PPARA, APOA1, LPL, CD36, ANGPTL4. The insulin resistance having the same negative log as the previous pathway include 5 out of 9 light green nodes (NOS3, SLC2A4, PPARGC1A, PPARA, CD36) distributed in the second cluster-ID. Having a negative log of -6.41 , 5 out of 10 brown nodes (OXTR, EDNRA, F2R, GHSR, PIK3R1) represent the cAMP signaling pathway located in the first cluster-ID. The adipocytokine signaling pathway having a negative log of -6.17 is composed of 7 turquoise nodes and among them, 5 nodes (SLC2A4, PPARGC1R, RXRA, PPARA, CD36) are within the second cluster ID. Having the same negative value as the previous one, the TNF signaling pathway included 8 gold nodes. The first set of 4 gold nodes (CCL2, PTGS2, TNF, NFKBIA) represented the fourth cluster-ID and the second set of 3 gold nodes (CASP3, EDN1, PIK3R1) is within the first cluster-ID. Finally, 5 out of 8 purple nodes (NCOA3, NCOA2, ESR1, ESR2, NOS3) represent the estrogen signaling pathway within the second cluster ID having a negative log of -5.62 . Overall, the top 25 KEGG pathways show various proportions between the negative $\log$ value and the gene ratio. For instance, the neuroactive ligand- 
receptor interaction, pathways in cancer, and the calcium signaling pathway represented as the top 3 KEGG pathways (Figure 4D) have a much higher negative log value compared to their gene ratio that falls below 0.10 . On the contrary, the cholesterol metabolism, the ovarian steroidogenesis, the PPAR signaling pathway, the adipocytokine signaling pathway, and the renin-angiotensin system have a gene ratio above 0.10
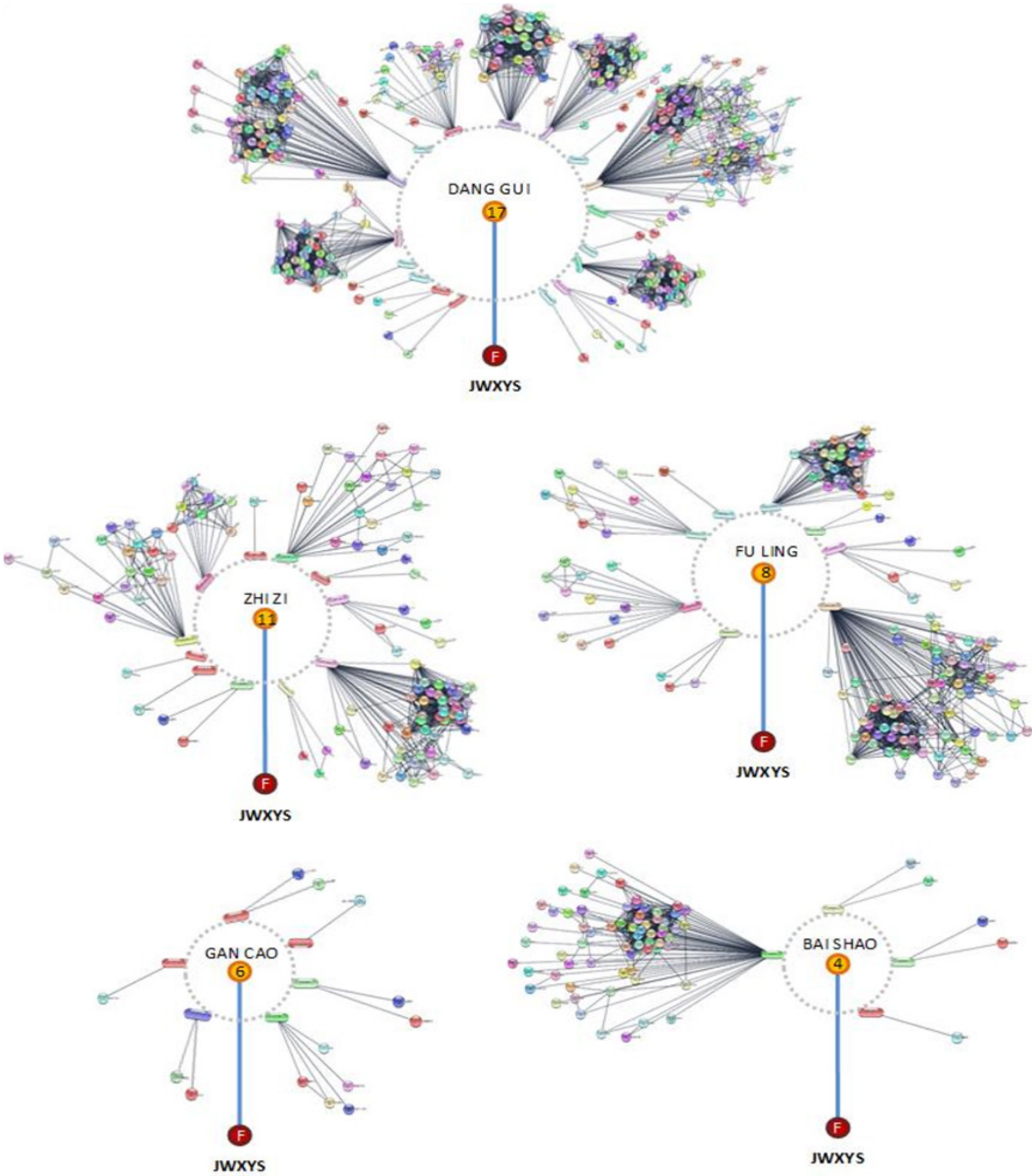

Citation: Leclerc B,Wu YC,Wu P.A network pharmacological approach to evaluate Jia-Wei-Xiao-Yao-san formula's mechanistic pathways and its implication in the symptomatology of polycystic ovarian syndrome. Int / Complement Alt Med. 2020; I3(5):200-2 I3. DOI: 10.15406/ijcam.2020.13.005 I7 


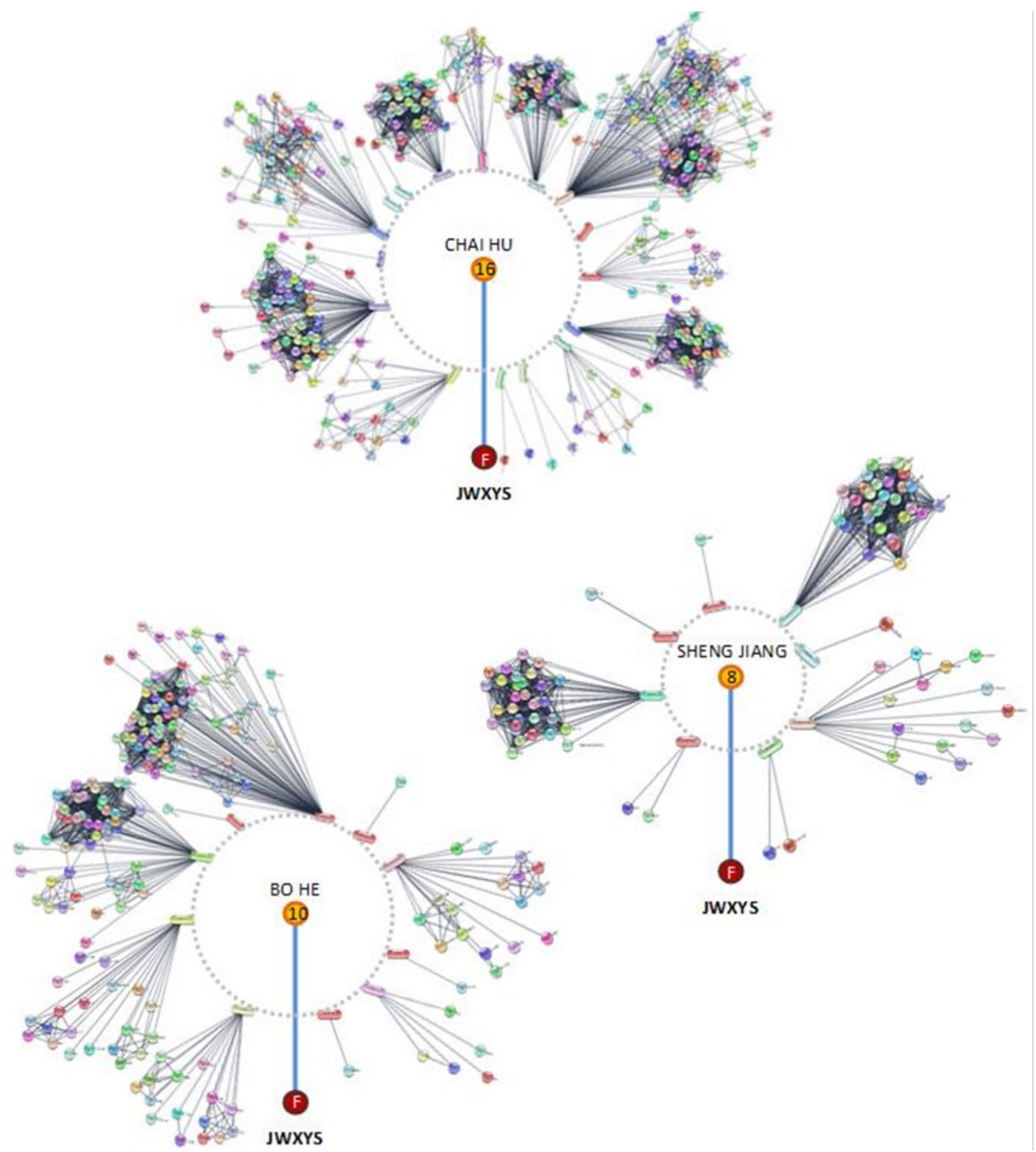

Figure 3 Representation of the highest interaction score to each ingredient among eight of ten single herbs composing JWXYS formula. In each herb, the highest interaction score of the compounds to PCOS-related targets are depicted along with the cross-binding interaction between targets. The diameter of the inner circle refers to the number of bioactive ingredients rotating around the circle of each herb. The herbs Bai Zhu and Mu Dan Pi are not illustrated here as their compounds are uncharacterized in the literature.

\section{Discussion}

Since women with PCOS have variable symptoms, western medicine does not always yield appreciable results. Therefore, determining the molecular pathways underlying the pathophysiology of PCOS is of the utmost importance. Here, we address the functional role of the JWXYS formula by developing a pharmacological approach that assesses the interaction scores between compounds or drugs with PCOS-related targets as well as the common targets shared by both CT and DT networks. 
According to our comparative analyses, the neuroactive ligandreceptor interaction pathway was ranked first with a score of $2.64 \mathrm{e}-15$ in the CDT network. Microarrays studies were conducted to evaluate differentially expressed genes behavior and how gene expression dataset with abnormal DNA methylation levels is associated with pathways in subcutaneous adipose tissue collected from women with PCOS. ${ }^{48,49}$ We did not see the same pool of genes in common with these two studies, except for the F2R gene which is involved in neuroactive ligand-receptor interaction, calcium signaling pathway as also reported by Liu et al., ${ }^{50}$ and also, we identified cAMP signaling pathway in our study. Interestingly, He et al. ${ }^{51}$ showed the neuroactive ligand-receptor interaction, PPAR signaling pathway, and cAMP signaling pathway were all involved in the development and progression of PCOS. Likewise, to our study, these pathways are listed among the top 10 highest scores. Since many PCOS patients also experience mental depression, studies mentioned that the neuroactive ligand-receptor interaction represents one of the core pathways modulating the major depressive disorder $^{52}$ and the premenstrual dysphoric disorder rat model,$^{53}$ and JWXYS formula is recognized to treat depression and premenstrual dysphoric disorder. ${ }^{36,40}$ Another study found that the up-regulation of the neuroactive ligand-receptor interaction may be a characteristic of premature ovarian failure (POF) ${ }^{54}$ Likewise, our study suggests that the cross-modulation of neuroactive ligand-receptor interaction along with the calcium and cAMP signaling pathways localized in the first cluster ID (red nodes) may explain their involvement in the development and progression of PCOS. Meanwhile, the popular consumption of JWXYS formula among Asian women suggests that its compounds strong interactions with 16 PCOS-related targets: PIK3R1, GNAQ, LPAR3, AGTR1, HTR2C, GNRHR, TACR3, GHSR, F2R, CHRM3, PTGER1, KISS1R, P2RY2, EDNRB, EDNRA, OXTR (Figure 4C) is associated with the neuroactive ligand-receptor interaction, calcium and cAMP signaling pathways, and upon their modulation, they alleviate chronic unpredictable mild stress, strengthen brain signaling circuits and, consequently, improve the depressive symptoms associated with PCOS, according to genetic studies findings. ${ }^{55-57}$
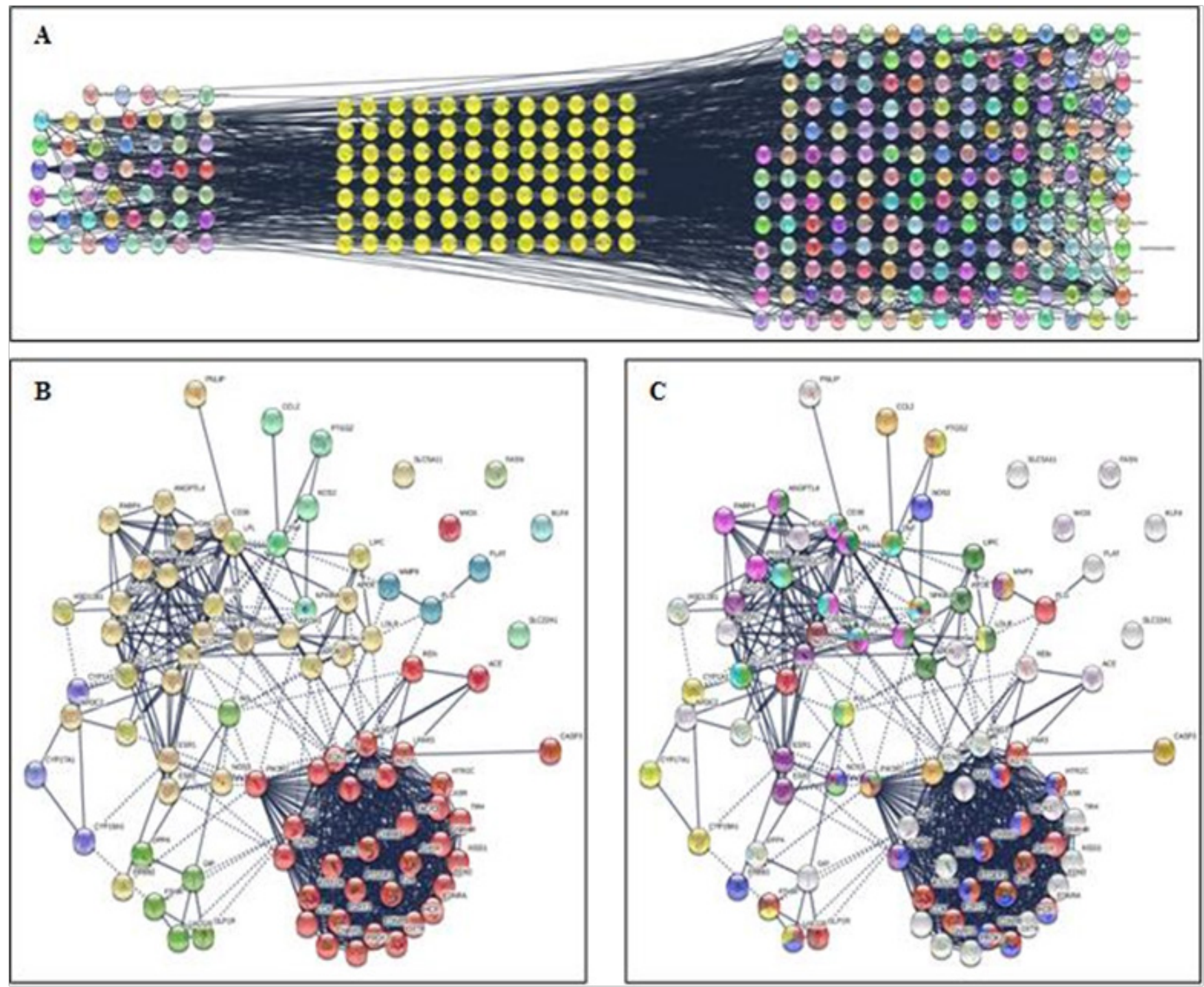

Citation: Leclerc B,Wu YC,Wu P.A network pharmacological approach to evaluate jia-Wei-Xiao-Yao-san formula's mechanistic pathways and its implication in the symptomatology of polycystic ovarian syndrome. Int J Complement Alt Med. 2020;13(5):200-213. DOI: I0.I5406/ijcam.2020.13.005I7 


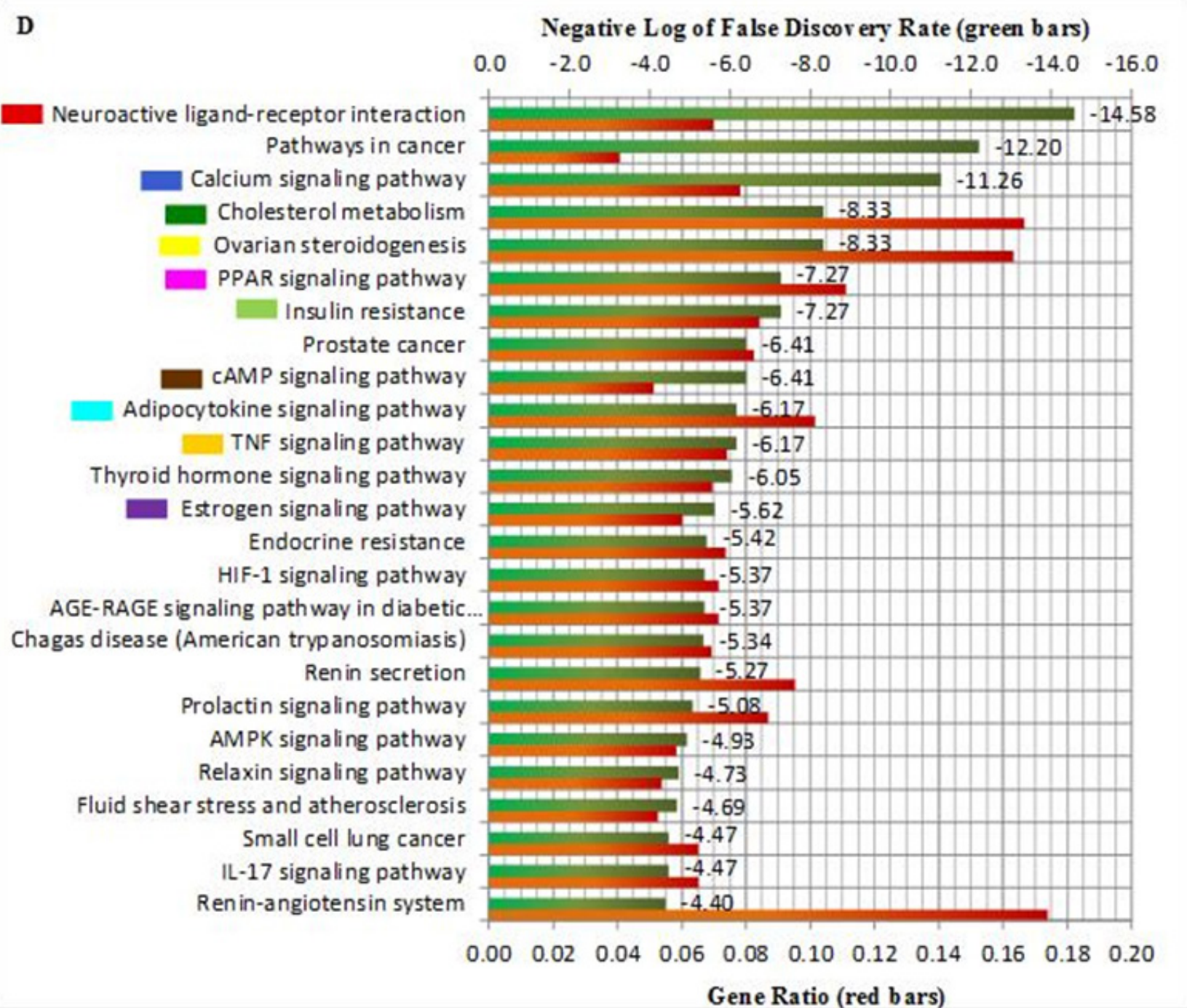

Figure 4 PCOS-related targets of JWXYS formula and drugs-treating PCOS. A) On the right panel, 190 PCOS-related genes were unique to the JWXYS formula, and on the left panel 49 PCOS-related targets were unique to the drugs-treating PCOS where nodes are labeled with arbitrary colors. PCOS-related targets, nodes labeled yellow, were common to both drugs and JWXYS formula. B) Clustering network of common compounds-drugs-PCOS-related targets. C) The highest score of KEGG pathways which are represented by nodes labeled with colors are compared with the clustering network of compounds-drugsPCOS-related targets. D) Histogram showing the top 25 KEGG pathways after analyzing the common compounds-drugs-PCOS-related targets. Colored rectangles at the left side of 10 KEGG pathways refer to the nodes' color depicted in figure C.

The estrogen signaling pathway identified in the CDT network retrieved the PCOS-related targets PIK3R1 and GNAQ in the first cluster-ID and ESR1, ESR2, NCOA2, NCOA3, NOS3, MMP9 in the second cluster ID (Figures $4 \mathrm{~B} \& 4 \mathrm{C}$ ). The induction of estrogen is important in the process of folliculogenesis and oocyte maturation resulting in ovulation. However, the estrogen signaling pathway may be disturbed and contribute to the development of PCOS and premature ovarian failure. The link between the estrogen signaling pathway and ovarian steroidogenesis may be explained at the JWXYS formula compounds mimicking miR-132 to induce the expression of CYP19A1 which increases the estrogen synthesis in mouse granulosa cells, ${ }^{58}$ and then estrogen could interact with the PCOS-related target ESR1 to activate the estrogen signaling pathway (Figures 4C \& 4D).
A similar study has shown that A-kinase anchor protein 95 is involved in modulating FSH to induce CYP19A1 expression and estrogen synthesis which A-kinase anchor protein 95 and CYP19A1 are low in human luteinizing granulosa cells collected from PCOS patients. ${ }^{59}$ At least in part, the JWXYS formula compounds interact with CYP19A1. The CDT network further indicates the JWXYS formula compounds could participate in modulating FSHR and LHCGR, therefore mimicking the endogenous FSH involved in stimulating the cAMP pathway in granulosa cells which then could regulate estrogen production, ${ }^{60}$ through the link activation of CYP19A1-ESR1 (Figure 4C). Another endocrine pathway shows that LH increases estradiol secretion in preantral follicles through up-regulating CYP17A1 and CYP19A1 gene transcripts in PCOS, ${ }^{61,62}$ and reflect those two nodes 
observed in the CDT network. In PCOS, chronic LH stimulation could impair FSH-dependent antral follicle growth via reducing FSHR expression in granulosa cells, ${ }^{61}$ a response that may be counteracted/ reverse by the JWXYS formula compounds. Also, the implication of JWXYS formula in up-regulating estrogen signaling pathway via ESR1 and ESR2 is known to act agonistically in the same line as the endogenous estrogen secretion does to decrease circulating LH, regularize the menstrual cycle period, and down-regulate PIK3R1 gene expression, ${ }^{63}$ as well to stimulate thecal interstitial cells, secondary follicle population, LHR and CYP17A1 gene transcript. ${ }^{62}$ The crosstalk between the estrogen signaling pathway and ovarian steroidogenesis is linked to PCOS. In our study, the ovarian steroidogenesis has shown 8 PCOS-related targets namely CYP17A1, CYP19A1, CYP1A1, FSHR, LHCGR, INS, LDLR, PTGS2. Their mechanisms of action are most likely related to insulin resistance. A human granulosaluteal cell culture study stimulated with metformin enhanced the insulin-stimulated translocation of glucose transporter-4 expression in the plasma membrane of granulosa cells which increased glucose uptake and the metabolism in those cells, therefore, inducing follicle growth in insulin resistance PCOS subjects. ${ }^{64}$ Interestingly, INS is interacting with PIK3R1, a key PCOS-related target, and suggests the involvement of crosstalk between the first and second cluster ID, i.e. between the neuroactive ligand-receptor interaction-calcium-cAMP signaling and insulin resistance-ovarian steroidogenesis. It has also been found that LHCGR overexpression in thecal cells of PCOS may be associated with higher CYP17A1 expression in antral follicles in PCOS compared to control ovaries. ${ }^{65}$ Granulosa cells in small antral follicles of PCOS patients indicated a higher LHCGR and CYP19A1 gene expression, but lower FSHR. ${ }^{66}$ Thecal cells of normal patients showed an increased level of LHCGR and CYP17A1 to facilitate folliculogenesis ${ }^{67}$ indicating that JWXYS formula compounds could have a constitutive role in regulating thecal and granulosa cells against the pathophysiology of PCOS. There have been other studies that point out the role of GIP that moderately induced FSHR gene transcript in granulosa cells ${ }^{68}$ as this node belongs to the third cluster ID and is shown in Figure 4C interacting with INS, LHCGR, and GLP1R, the latter receptor involved with the gut hormone GLP-1 which is impaired in obese women with PCOS and when stimulated by GLP-1 it can have a positive effect on the body weight, serum testosterone levels and even the polycystic ovarian morphology. ${ }^{69-71}$ The interaction between GIP and GLP1R is currently unknown in PCOS subjects, but GLP1R agonists have been clinically reported in the treatment of type 2 diabetes and obesity, ${ }^{72}$ and since JWXYS formula compounds interact with GLP1R and support why the PCOS subjects express higher insulin and total GIP levels and lower GLP-1 levels in a late phase of oral glucose tolerance test when compared with healthy women control. ${ }^{73}$

Although little evidence points out TNF signaling pathway crosstalk with the PPAR signaling pathway, ${ }^{74,75}$ further research should explore the relationship between high inflammatory reaction and the severity of the PCOS conditions. Insofar, TNF alpha is higher in PCOS women, ${ }^{76}$ and to inhibit FSH-induced follicle development, estradiol secretion and ovulation rate and a restorative effect may be acting through the PPARG pathway in mouse preantral follicles. ${ }^{74}$

The crosstalk between multiple pathways plays an essential role in regulating several biological processes during cell-to-cell interactions. Sequentially, CD36 interacts with PPAR which interacts with NFKBIA and in turn interacts with PIK3R1. These four intersecting PCOS-related targets are important regulators of multiple signaling pathways. For instances, CD36 is involved in cholesterol metabolism, insulin resistance, adipocytokine, and PPAR signaling pathways; PPAR is involved in insulin resistance, PPAR, cAMP, and adipocytokine signaling pathways; NFKBIA is involved in insulin resistance, adipocytokine, cAMP, and TNF signaling pathways; and finally PIK3R1 is involved in insulin resistance, cAMP, estrogen, and TNF signaling pathways. Notably, insulin resistance is the only pathway involving the four PCOS-related targets CD36, NFKBIA, PIK3R1, PPARA, and each of them is implicated, at least, in three other pathways. Therefore, insulin resistance is intersecting with other signaling pathways, mainly with adipocytokine and cAMP signaling pathways. The other PCOS-related targets NOS3, SLC2A4, PPARGC1A, TNF, INS are also known to participate in the mechanism of insulin resistance (Figure 4C). According to Seow et al., ${ }^{77}$ the protein level of CD36 and hormone-sensitive lipase were significantly higher and lower respectively in PCOS women than in healthy control. Given those observations, CD36 and LPL share the same cholesterol metabolism and PPAR signaling pathway, but no significant interaction exists with LPL. However, LPL does interact with LIPC as a hepatic enzyme involved in fat hydrolysis. Another study has observed, at the baseline, an up-regulation of insulin resistance genes: NFKBIA, PPARA, and PPARGC1A involved in PCOS which may be an effect mediated via insulin sensitivity, ${ }^{78,79}$ as we observe sequential interactions NFKBIA to PPARA and PPARA to PPARGC1A (Figure 4C). In contrast to the PPARGC1A polymorphism associated with the PCOS susceptibility, the relationship between PIK3R1, SLC2A4, and NOS3 variants with the occurrence of PCOS or in post-insulin receptor signaling in PCOS subjects was not significant; ${ }^{80,81}$ these three PCOS-related targets were not interacting with each other (Figure 4C). However, it has been found that the transcript of NOS3 in PCOS peripheral blood is reduced may be due as a consequence of the system to increase catalase levels to prevent the oxidative built-up in the body. ${ }^{82}$ As the interaction between TNF and INS exists in Figure $4 \mathrm{C}$, there may be an implication of TNF polymorphism associated with PCOS development, but the high serum levels of TNF-alpha were definitively increased in PCOS patients. ${ }^{83,84}$ TNFalpha is especially high in obesity-PCOS compared with normalweight female subjects. ${ }^{85}$ The different mechanisms modulated by TNF-alpha and IL-6 negatively affect insulin signaling, via decreased adiponectin signaling and glucose transporter type 4 protein, which effect is exacerbated in the endometrium of obese PCOS women leading to an abnormal endometrial response to androgen and insulin stimulation and consequently leading to a reproductive failure. ${ }^{85,86}$ They demonstrated the active form of insulin receptor substrate 1 was declined by TNF-alpha while the inactive form was increased by IL-6 in obese PCOS. With this perspective in mind, the formula compounds may interact with PCOS-related targets TNF and INS to counteract the effect of hyperandrogenic and hyperinsulinemic conditions over glucose uptake in the endometrium causing receptivity failure in obese PCOS subjects.

\section{Conclusion}

Our related-drug model proposed in this study has strengthened the findings that the JWXYS formula is an appropriate TCM to treat at least the women symptoms associated with PCOS manifestation. The multiple pathways JWXYS formula modulates underlie its numerous bioactive compounds interacting with PCOS-related targets. Most of the pathways we have identified are well documented in the 
literature, and the intersecting pathways incited by JWXYS formula compounds reveals its multifaceted biological effects to improve the PCOS-related characteristics, such as having a role in FSH-dependent estrogen production in granulosa cells, lowering luteinizing hormone, improving the bodyweight via adipocyte disposition in inguinal and mesenteric fat areas, alleviating insulin resistance, and regulating ovarian gene expression. The compounds-drugs interactions with PCOS-related targets reveal that ovarian steroidogenesis, PPAR, and adipocytokine signaling pathways are among those KEGG pathways with a gene ratio $\geq 0.10$. The gene ratio value may indicate the signaling pathways trends preferentially regulated in the CDT network. Further experimental research is sought to validate the molecular mechanisms by which the JWXYS formula alleviates the symptoms of PCOS women. Thus, the pharmacological network methodology employed in this study demonstrates the feasibility showing the functional interactions between the compounds composing JWXYS formula and the gene targets validated in PCOS patients, the common PCOSrelated targets found between the compounds and related-drugs.

\section{Acknowledgments}

BL contributed to the pharmacological network analyses regarding the Jia-Wei-Xiao-Yao-San formula. PW contributed to the literature review and topic direction in polycystic ovarian syndrome. YCW contributed discussion and trends about herbal medicine principles implicated in women's gynecology and health. BL and PW would like to address special thanks to YCW for his kindness in sharing inspirational ideas involved in traditional herbal medicine.

\section{Conflict of interests}

The authors declare that they have no competing interests.

\section{Funding}

There is no funding declaration to report from third party.

\section{References}

1. Bozdag G, Mumusoglu S, Zengin D, et al. The prevalence and phenotypic features of polycystic ovary syndrome: a systematic review and meta-analysis. Hum Reprod. 2016;31(12):2841-2855

2. Skiba MA, Islam RM, Bell RJ, et al. Understanding variation in prevalence estimates of polycystic ovary syndrome: a systematic review and meta-analysis. Hum Reprod Update. 2018;24(6):694-709.

3. Kim JJ, Choi YM. Phenotype and genotype of polycystic ovary syndrome in Asia: Ethnic differences. J Obstet Gynaecol Res. 2019;45(12):2330-2337.

4. Pasquali R, Gambineri A. A comprehensive approach in diagnosing the polycystic ovary syndrome. Womens Health (Lond). 2015;11(4):501512

5. LiY, Wei LN, Xiong YL, et al. Effect of luteinizing hormone vs follicular stimulating hormone ratio on anti-Müllerian hormone secretion and folliculogenesis in patients with polycystic ovarian syndrome. Zhonghua Fu Chan Ke Za Zhi. 2010;45(8):567-570.

6. Bozkurt Koseoglu S, Dinc Elibol F. Does the pituitary gland volume change in polycystic ovary syndrome? Gynecol Obstet Invest. 2018;83(5):515-519.

7. Burt Solorzano CM, Beller JP, Abshire MY, et al. Neuroendocrine dysfunction in polycystic ovary syndrome. Steroids. 2012;77(4):332337.
8. Legro RS. Evaluation and treatment of polycystic ovary syndrome: Endocrinology of female reproduction. Endotext. 2017.

9. Amato MC, Verghi M, Nucera M, et al. Low estradiol-to-testosterone ratio is associated with oligo-anovulatory cycles and atherogenic lipidic pattern in women with polycystic ovary syndrome. Gynecol Endocrinol. 2011;27(8):579-586.

10. Paixao L, Ramos RB, Lavarda A, et al. Animal models of hyperandrogenism and ovarian morphology changes as features of polycystic ovary syndrome: a systematic review. Reprod Biol Endocrinol. 2017;15(1):12.

11. Saddick SY. Identifying genes associated with the development of human polycystic ovary syndrome. Saudi J Biol Sci. 2020;27(5):12711279 .

12. Goodarzi MO, Carmina E, Azziz R. DHEA, DHEAS and PCOS. $J$ Steroid Biochem Mol Biol. 2015;145:213-225.

13. Moran C, Arriaga M, Arechavaleta-Velasco F, et al. Adrenal androgen excess and body mass index in polycystic ovary syndrome. J Clin Endocrinol Metab. 2015;100(3):942-950.

14. Dumesic DA, Richards JS. Ontogeny of the ovary in polycystic ovary syndrome. Fertil Steril. 2013;100(1):2338 .

15. Li W, Li Q. Dysregulation of glucose metabolism even in Chinese PCOS women with normal glucose tolerance. Endocr J. 2012;59(9):765-770.

16. Malin SK, Kirwan JP, Sia CL, et al. Pancreatic $\beta$-cell dysfunction in polycystic ovary syndrome: role of hyperglycemia-induced nuclear factor- $\mathrm{\kappa B}$ activation and systemic inflammation. Am J Physiol Endocrinol Metab. 2015;308(9):E770-E777.

17. Zhang B, Wang J, Shen S, et al. Association of androgen excess with glucose intolerance in women with polycystic ovary syndrome. Biomed Res Int. 2018;2018:6869705.

18. Cree-Green M, Cai N, Thurston JE, et al. Using simple clinical measures to predict insulin resistance or hyperglycemia in girls with polycystic ovarian syndrome. Pediatr Diabetes. 2018;19(8):1370 1378 .

19. Wang F, Zhang ZH, Xiao KZ, et al. Roles of hypothalamic-pituitaryadrenal axis and hypothalamus-pituitary-ovary axis in the abnormal endocrine functions in patients with polycystic ovary syndrome. Zhongguo Yi Xue Ke Xue Yuan Xue Bao. 2017;39(5):699-704.

20. Sanchez-Garrido MA, Tena-Sempere M. Metabolic dysfunction in polycystic ovary syndrome: Pathogenic role of androgen excess and potential therapeutic strategies. Mol Metab. 2020;35:100937.

21. Zhu JL, Chen Z, Feng WJ, et al. Sex hormone-binding globulin and polycystic ovary syndrome. Clin Chim Acta. 2019;499:142-148.

22. Holte J. Disturbances in insulin secretion and sensitivity in women with the polycystic ovary syndrome. Baillieres Clin Endocrinol Metab. 1996;10(2):221-47.

23. Pinola $\mathrm{P}$, Lashen $\mathrm{H}$, Bloigu A, et al. Menstrual disorders in adolescence: a marker for hyperandrogenaemia and increased metabolic risks in later life? Finnish general population-based birth cohort study. Hum Reprod. 2012;27(11):3279-3286.

24. Dumesic DA, Oberfield SE, Stener-Victorin E, et al. Scientific statement on the diagnostic criteria, epidemiology, pathophysiology, and molecular genetics of polycystic ovary syndrome. Endocr Rev. 2015;36(5):487-525. 
25. Javed Z, Papageorgiou M, Deshmukh H, et al. A randomized, controlled trial of vitamin D supplementation on cardiovascular risk factors, hormones, and liver markers in women with polycystic ovary syndrome. Nutrients. 2019;11(1):188.

26. Ma RC, Liu KH, Lam PM, et al. Sonographic measurement of mesenteric fat predicts presence of fatty liver among subjects with polycystic ovary syndrome. $J$ Clin Endocrinol Metab. 2011;96(3):799-807.

27. de Medeiros SF, de Medeiros MAS, Barbosa BB, et al. The Role of visceral adiposity index as predictor of metabolic syndrome in obese and nonobese women with polycystic ovary syndrome. Metab Syndr Relat Disord. 2020.

28. Fernandez RC, Moore VM, Van Ryswyk EM, et al. Sleep disturbances in women with polycystic ovary syndrome: prevalence, pathophysiology, impact and management strategies. Nat Sci Sleep. 2018;10:45-64.

29. Thannickal A, Brutocao C, Alsawas M, et al. Eating, sleeping and sexual function disorders in women with polycystic ovary syndrome (PCOS): A systematic review and meta-analysis. Clin Endocrinol (Oxf). 2020;92(4):338-349.

30. Yin $\mathrm{X}$, Ji Y, Chan CLW, et al. The mental health of women with polycystic ovary syndrome: a systematic review and meta-analysis. Arch Womens Ment Health. 2020. 10.1007/s00737020-01043-X.

31. Jiskoot G, Dietz de Loos A, Beerthuizen A, et al. Long-term effects of a three-component lifestyle intervention on emotional well-being in women with polycystic ovary syndrome (PCOS): A secondary analysis of a randomized controlled trial. PLoS One. 2020;15(6):e0233876.

32. Teng Jialin. Chinese Materia Medica. $1^{\text {st }}$ edn. China: People's Medical Publishing House; 2007.

33. Chen JT, Shiraki M. Menopausal hot flash and calcitonin generelated peptide; effect of Keishi-bukuryo-gan, a kampo medicine, related to plasma calcitonin gene-related peptide level. Maturitas. 2003;45(3):199-204.

34. Yasui T, Matsui S, Yamamoto S, et al. Effects of Japanese traditional medicines on circulating cytokine levels in women with hot flashes. Menopause. 2011;18(1):85-92.

35. Terauchi M, Hiramitsu S, Akiyoshi M, et al. Effects of three Kampo formulae: Tokishakuyakusan (TJ-23), Kamishoyosan (TJ-24), and Keishibukuryogan (TJ-25) on Japanese peri- and postmenopausal women with sleep disturbances. Arch Gynecol Obstet. 2011;284(4):913-921

36. Park DM, Kim SH, Park YC, et al. The comparative clinical study of efficacy of Gamisoyo-San (Jiaweixiaoyaosan) on generalized anxiety disorder according to differently manufactured preparations: multicenter, randomized, double blind, placebo controlled trial. $J$ Ethnopharmacol. 2014;158 Pt A:11-7.

37. Chen H, Chan HC. Amplification of FSH signalling by CFTR and nuclear soluble adenylyl cyclase in the ovary. Clin Exp Pharmacol Physiol. 2017;44 Suppl 1:78-85.

38. Lin YR, Wu MY, Chiang JH, et al. The utilization of traditional Chinese medicine in patients with dysfunctional uterine bleeding in Taiwan: a nationwide population-based study. BMC Complement Altern Med. 2017;17(1):427.

39. Chen HY, Lin YH, Su IH, et al. Investigation on Chinese herbal medicine for primary dysmenorrhea: implication from a nationwide prescription database in Taiwan. Complement Ther Med. 2014;22(1):116-125.
40. Yamada K, Kanba S. Effectiveness of kamishoyosan for premenstrual dysphoric disorder: open-labeled pilot study. Psychiatry Clin Neurosci. 2007;61(3):323-325.

41. Kimura Y, Takamatsu K, Fujii A, et al. Kampo therapy for premenstrual syndrome: efficacy of Kamishoyosan quantified using the second derivative of the fingertip photoplethysmogram. J Obstet Gynaecol Res. 2007;33(3):325-332.

42. Yasui T, Yamada M, Uemura H, et al. Changes in circulating cytokine levels in midlife women with psychological symptoms with selective serotonin reuptake inhibitor and Japanese traditional medicine. Maturitas. 2009;62(2):146-152.

43. Ushiroyama T, Ikeda A, Sakuma K, et al. Changes in serum tumor necrosis factor (TNF-alpha) with kami-shoyo-san administration in depressed climacteric patients. Am J Chin Med. 2004;32(4):621-629.

44. Chen YL, Lee CY, Huang KH, et al. Prescription patterns of Chinese herbal products for patients with sleep disorder and major depressive disorder in Taiwan. J Ethnopharmacol. 2015;171:307-316.

45. Chen HY, Huang BS, Lin YH, et al. Identifying Chinese herbal medicine for premenstrual syndrome: implications from a nationwide database. BMC Complement Altern Med. 2014;14:206.

46. Lin MJ, Chen HW, Liu PH, et al. The prescription patterns of traditional Chinese medicine for women with polycystic ovary syndrome in Taiwan: A nationwide population-based study. Medicine (Baltimore). 2019;98(24):e15890

47. Radosh L. Drug treatments for polycystic ovarian syndrome. Am Fam Physician. 2009;79(8):671-766.

48. Zhang $\mathrm{CH}$, Ma K, Yuan BC, et al. Bushen Huoxue herbal medicine for treating hyperprolactinemia in women: a Meta-analysis. Zhongguo Zhong Yao Za Zhi. 2019;44(6):1087-1093

49. Yin XD, Xue XO, Wang JS, et al. Effect of Bushen Huoxue recipe on women with thin endometrial ovulation disorder and a rat model of thin endometrium resulted from kidney deficiency-related blood stasis. Gynecol Endocrinol. 2020:1-5.

50. Liu L, He D, Wang Y, et al. Integrated analysis of DNA methylation and transcriptome profiling of polycystic ovary syndrome. Mol Med Rep. 2020;21(5):2138-2150.

51. He D, Liu L, Wang Y, et al. A Novel Genes Signature Associated with the Progression of Polycystic Ovary Syndrome. Pathol Oncol Res. 2020;26(1):575-582.

52. Liu Y, Fan P, Zhang S, et al. Prioritization and comprehensive analysis of genes related to major depressive disorder. Mol Genet Genomic Med. 2019;7(6):e659.

53. Wei S, Sun P, Guo Y, et al. Gene Expression in the Hippocampus in a Rat Model of Premenstrual Dysphoric Disorder After Treatment With Baixiangdan Capsules. Front Psychol. 2018;9:2065.

54. Lin J, Zheng J, Zhang H, et al. Cytochrome P450 family proteins as potential biomarkers for ovarian granulosa cell damage in mice with premature ovarian failure. Int J Clin Exp Pathol. 2018;11(8):42364246.

55. Sun X, Song Z, Si Y, et al. microRNA and mRNA profiles in ventral tegmental area relevant to stress-induced depression and resilience. Prog Neuropsychopharmacol Biol Psychiatry. 2018;86:150-165.

56. Liu Y, Fan P, Zhang S, et al. Prioritization and comprehensive analysis of genes related to major depressive disorder. Mol Genet Genomic Med. 2019;7(6):e659. 
57. Zhang Y, Li M, Wang Q, et al. A joint study of whole exome sequencing and structural MRI analysis in major depressive disorder. Psycho Med. 2020;50(3):384-395.

58. Wu S, Sun H, Zhang Q, et al. MicroRNA-132 promotes estradio synthesis in ovarian granulosa cells via translational repression of Nurr1. Reprod Biol Endocrinol. 2015;13:94.

59. $\mathrm{Gu} \mathrm{Y,} \mathrm{Xu} \mathrm{W,} \mathrm{Zhuang} \mathrm{B,} \mathrm{et} \mathrm{al.} \mathrm{Role} \mathrm{of} \mathrm{A-kinase} \mathrm{anchoring} \mathrm{protein} 95$ in the regulation of cytochrome P450 family 19 subfamily A member 1 (CYP19A1) in human ovarian granulosa cells. Reprod Fertil Dev. 2018;30(8):1128-3116.

60. Chen H, Chan HC. Amplification of FSH signalling by CFTR and nuclear soluble adenylyl cyclase in the ovary. Clin Exp Pharmaco Physiol. 2017;44 Suppl 1:78-85.

61. Orisaka M, Hattori K, Fukuda S, et al. Dysregulation of ovarian follicular development in female rat: LH decreases FSH sensitivity during preantral-early antral transition. Endocrinology. 2013;154(8):2870 2880 .

62. Marcondes RR, Carvalho KC, Duarte DC, et al. Differences in neonatal exposure to estradiol or testosterone on ovarian function and hormonal levels. Gen Comp Endocrinol. 2015;212:28-33.

63. Maliqueo M, Benrick A, Alvi A, et al. Circulating gonadotropins and ovarian adiponectin system are modulated by acupuncture independently of sex steroid or $\beta$-adrenergic action in a female hyperandrogenic rat model of polycystic ovary syndrome. Mol Cell Endocrinol. 2015;412:159-169.

64. Rice S, Pellatt LJ, Bryan SJ, et al. Action of metformin on the insulinsignaling pathway and on glucose transport in human granulosa cells. $J$ Clin Endocrinol Metab. 2011;96(3):E427-E435.

65. Comim FV, Teerds K, Hardy K, et al. Increased protein expression of LHCG receptor and 17 $\alpha$-hydroxylase/17-20-lyase in human polycystic ovaries. Hum Reprod. 2013;28(11):3086-3092.

66. Owens LA, Kristensen SG, Lerner A, et al. Gene Expression in Granulosa Cells From Small Antral Follicles From Women With or Without Polycystic Ovaries. J Clin Endocrinol Metab. 2019;104(12):6182-6192.

67. Asiabi P, Leonel ECR, Marbaix E, et al. Immunodetection and quantification of enzymatic markers in theca cells: the early process of ovarian steroidogenesis. Biol Reprod. 2020;102(1):145-155.

68. Nishiyama $\mathrm{Y}$, Hasegawa $\mathrm{T}$, Fujita $\mathrm{S}$, et al. Incretins modulate progesterone biosynthesis by regulating bone morphogenetic protein activity in rat granulosa cells. J Steroid Biochem Mol Biol. 2018;178:82-88.

69. Tzotzas T, Karras SN, Katsiki N. Glucagon-Like Peptide-1 (GLP-1) Receptor Agonists in the Treatment of Obese Women with Polycystic Ovary Syndrome. Curr Vasc Pharmacol. 2017;15(3):218-229.

70. Frossing S, Nylander M, Chabanova E, et al. Effect of liraglutide on ectopic fat in polycystic ovary syndrome: A randomized clinical trial. Diabetes Obes Metab. 2018;20(1):215-218.

71. Jensterle M, Janez A, Fliers E, et al. The role of glucagon-like peptide-1 in reproduction: from physiology to therapeutic perspective. Hum Reprod Update. 2019;25(4):504-517.

72. Hansen MSS, Tencerova M, Frølich J, et al. Effects of gastric inhibitory polypeptide, glucagon-like peptide-1 and glucagon-like peptide-1 receptor agonists on Bone Cell Metabolism. Basic Clin Pharmacol Toxicol. 2018;122(1):25-37.
73. Vrbikova J, Hill M, Bendlova B, et al. Incretin levels in polycystic ovary syndrome. Eur J Endocrinol. 2008;159(2):121127.

74. Hara S, Takahashi T, Amita M, et al. Bezafibrate restores the inhibition of FSH-induced follicular development and steroidogenesis by tumor necrosis factor-alpha through peroxisome proliferatoractivated receptor-gamma pathway in an in vitro mouse preantral follicle culture. Biol Reprod. 2011;85(5):895-906.

75. D Prabhu Y, Valsala Gopalakrishnan A. $\gamma$-Linolenic acid ameliorates DHEA induced pro-inflammatory response in polycystic ovary syndrome via PPAR $-\gamma$ signaling in rats. Reprod Biol. 2020;20(3):348-356

76. Cardoso NS, Ribeiro VB, Dutra SGV, et al. Polycystic ovary syndrome associated with increased adiposity interferes with serum levels of TNF-alpha and IL-6 differently from leptin and adiponectin. Arch Endocrinol Metab. 2020;64(1):4-10.

77. Seow KM, Tsai YL, Hwang JL, et al. Omental adipose tissue overexpression of fatty acid transporter CD36 and decreased expression of hormone-sensitive lipase in insulin-resistant women with polycystic ovary syndrome. Hum Reprod. 2009;24(8):19821988.

78. San-Millan JL, Escobar-Morreale HF. The role of genetic variation in peroxisome proliferator-activated receptors in the polycystic ovary syndrome (PCOS): an original case-control study followed by systematic review and meta-analysis of existing evidence. Clin Endocrinol (Oxf). 2010;72(3):383-392.

79. Dantas WS, Murai IH, Perandini LA, et al. Acute exercise elicits differential expression of insulin resistance genes in the skeletal muscle of patients with polycystic ovary syndrome. Clin Endocrinol (Oxf). 2017;86(5):688-697.

80. Jones MR, Wilson SG, Mullin BH, et al. Polymorphism in postinsulin receptor signaling pathway is not associated with polycystic ovary syndrome. Fertil Steril. 2008;90(6):2298-2303.

81. Walch K, Kolbus A, Hefler-Frischmuth K. Polymorphisms of the endothelial nitric oxide synthase gene in premenopausal women with polycystic ovary syndrome. Maturitas. 2008;61(3):256-259.

82. Krishna MB, Joseph A, Thomas PL, et al. Impaired Arginine Metabolism Coupled to a Defective Redox Conduit Contributes to Low Plasma Nitric Oxide in Polycystic Ovary Syndrome. Cell Physiol Biochem. 2017;43(5):1880-1892.

83. de Alencar JB, Alves HV, Elpidio LN, et al. Polymorphisms of Cytokine Genes and Polycystic Ovary Syndrome: A Review. Metab Syndr Relat Disord. 2016;14(10):468-74

84. Kordestani F, Mazloomi S, Mortazavi Y, et al. Preliminary study showing no association between G238A (rs361525) tumor necrosis factor- $\alpha(\mathrm{TNF}-\alpha)$ gene polymorphism and its serum level, hormonal and biochemical aspects of polycystic ovary syndrome. BMC Med Genet. 2018;19(1):149.

85. Orostica L, Poblete C, Romero C, et al. Pro-Inflammatory Markers Negatively Regulate IRS1 in Endometrial Cells and Endometrium from Women with Obesity and PCOS. Reprod Sci. 2020;27(1):290300

86. Orostica L, Garcia P, Vera C, et al. Effect of TNF- $\alpha$ on Molecules Related to the Insulin Action in Endometrial Cells Exposed to Hyperandrogenic and Hyperinsulinic Conditions Characteristics of Polycystic Ovary Syndrome. Reprod Sci. 2018;25(7):1000-1009. 